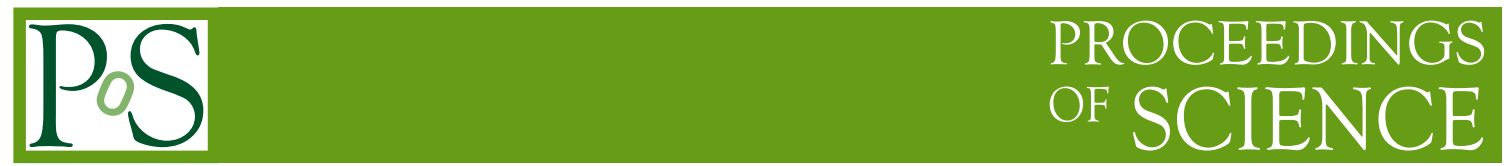

\title{
An extensive study of dark matter and neutrino phenomenology in the Triplet + Singlet Scotogenic Model
}

\author{
Ivania M. Ávila ${ }^{a, *}$ \\ ${ }^{a}$ Instituto de Física, Pontificia Universidad Católica de Chile, \\ Vicuña Mackenna 4860, Santiago de Chile - Chile \\ E-mail: idmaturana@uc.cl
}

In this proceeding, I will address the possibility that the nature of dark matter is associated with neutrino mass generation. Focusing on an extension of the Standard Model where the light neutrino masses are generated radiatively, we study the properties of a dark matter candidate which is made stable by the same symmetry responsible for the radiative origin of neutrino masses. Also, the model studied proposes a dark matter candidate that could be both a neutral scalar or a fermion. I will discuss the phenomenology of both scenarios, studying the parameter space which allows to reproduce the observed dark matter abundance. I will also comment on the expected signals in direct detection experiments, via indirect detection probes and at colliders.

40th International Conference on High Energy physics - ICHEP2020

July 28 - August 6, 2020

Prague, Czech Republic (virtual meeting)

\footnotetext{
${ }^{*}$ Speaker
} 
Introduction An extension of the scotogenic model [1] was proposed in [2]. In addition to the SM gauge symmetry there is a discrete $\mathbb{Z}_{2}$ symmetry that makes the lightest $\mathbb{Z}_{2}$-odd or "dark" particle stable and to ensure the radiative generation of neutrino masses. In this model, two neutrinos acquire mass and this will have implications on $0 v \beta \beta$ process [3]. The full particle content of the model is given in Table 1. Taking into account the new fields and symmetries of the model, the relevant terms of the Lagrangian read

$$
\begin{aligned}
\mathcal{L} & \subset-Y^{\alpha \beta} L_{\alpha} e_{\beta} \phi-Y_{F}^{\alpha}\left(\bar{L}_{\alpha} \tilde{\eta}\right) F-Y_{\Sigma}^{\alpha} \bar{L}_{\alpha}^{c} \Sigma^{\dagger} \tilde{\eta}-Y_{\Omega} \operatorname{Tr}[\bar{\Sigma} \Omega] F \\
& -\frac{1}{2} M_{\Sigma} \operatorname{Tr}\left(\bar{\Sigma}^{c} \Sigma\right)-\frac{M_{F}}{2} \overline{F^{c}} F+\text { h.c. }
\end{aligned}
$$

where $\tilde{\eta}=i \sigma_{2} \eta^{*}$. The first Yukawa term $Y^{\alpha \beta}$ is the SM interaction for leptons, which we can assume to be diagonal in flavor (Greek indices stand for family indices). More information about the scalar potential and the fermionic sector of this model is shown in [3]. For the scalar sector, the physical masses will be

$$
\begin{aligned}
m_{H^{ \pm}}^{2} & =\mu_{1} \frac{\left(v_{\phi}^{2}+2 v_{\Omega}^{2}\right)}{2 v_{\Omega}}, \\
m_{\eta^{ \pm}}^{2} & =m_{\eta}^{2}+\frac{1}{2} \lambda_{3} v_{\phi}^{2}+\frac{1}{\sqrt{2}} \mu_{2} v_{\Omega}+\frac{1}{2} \lambda_{\eta}^{\Omega} v_{\Omega}^{2} . \\
m_{\eta_{R}}^{2} & =m_{\eta}^{2}+\frac{1}{2}\left(\lambda_{3}+\lambda_{4}+\lambda_{5}\right) v_{\phi}^{2}+\frac{1}{2} \lambda_{\eta}^{\Omega} v_{\Omega}^{2}-\frac{1}{\sqrt{2}} \mu_{2} v_{\Omega}, \\
m_{\eta_{I}}^{2} & =m_{\eta}^{2}+\frac{1}{2}\left(\lambda_{3}+\lambda_{4}-\lambda_{5}\right) v_{\phi}^{2}+\frac{1}{2} \lambda_{\eta}^{\Omega} v_{\Omega}^{2}-\frac{1}{\sqrt{2}} \mu_{2} v_{\Omega} .
\end{aligned}
$$

In this work we will assume the $\mathbb{Z}_{2}$-odd scalar $\eta_{R}$ to be the dark matter candidate. The dark matter sector has therefore common features with the simplest scotogenic constructions [1] as well as the Inert Higgs Doublet Model [4-6].

\begin{tabular}{|c|r|r|r|r|r|r|r|}
\hline & \multicolumn{2}{|c|}{ Standard Model } & \multicolumn{2}{c|}{ new fermions } & \multicolumn{2}{c|}{ new scalars } \\
\hline & $L$ & \multicolumn{1}{c|}{$e$} & \multicolumn{1}{c|}{$\phi$} & \multicolumn{1}{c|}{$\Sigma$} & $F$ & $\eta$ & $\Omega$ \\
\hline Generations & 3 & 3 & 1 & 1 & 1 & 1 & 1 \\
\hline \hline $\mathrm{SU}(3)_{\mathrm{C}}$ & 1 & 1 & 1 & 1 & 1 & 1 & 1 \\
$\mathrm{SU}(2)_{\mathrm{L}}$ & 2 & 1 & 2 & 3 & 1 & 2 & 3 \\
$\mathrm{U}(1)_{\mathrm{Y}}$ & -1 & -2 & 1 & 0 & 0 & 1 & 0 \\
$\mathbb{Z}_{2}$ & + & + & + & - & - & - & + \\
\hline $\mathrm{L}$ & 1 & 1 & 0 & 0 & 0 & -1 & 0 \\
\hline
\end{tabular}

Table 1: Particle content and quantum numbers of the Singlet + Triplet Scotogenic Model.

Scalar dark matter results For our analysis we considered the constraints detailled in [3]. They include lepton flavor violation, neutrino oscillations, the invisible decay of the Higgs, as well as constraints from cosmology and dark matter phenomenology, explained in the following.

Fig. 1 depicts the expected dark matter relic abundance as a function of the mass of the scalar dark matter candidate $\eta_{R}$. Cyan points fall within the $3 \sigma$ C.L. cold dark matter measurement 


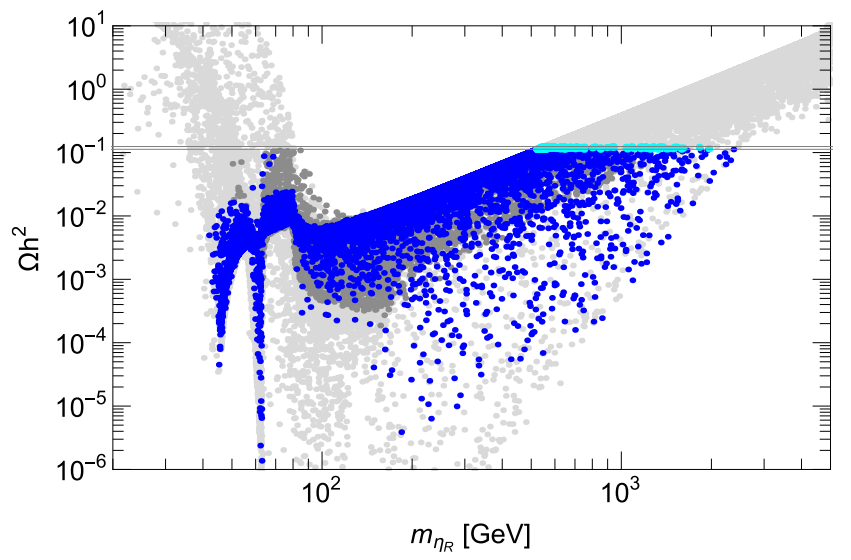

Figure 1: Relic abundance $\Omega_{\eta_{R}} h^{2}$ as a function of the $\eta_{R}$ mass.

by the Planck collaboration [7] explain the totality of dark matter in the Universe. Blue points refer to solutions where $\eta_{R}$ would be subdominant, and another dark matter candidate would be required. Grey points are instead excluded by any constraints, except the one by the current limit on WIMP-nucleon spin independent elastic scattering cross section set by XENON1T [8], represented by dark grey points. The features appearing in the plot can be explained by looking in detail into the $\eta_{R}$ annihilation channels. It is important to considered that also coannihilations with $\eta_{I}$ and $\eta^{ \pm}$ may occur in all regions of the parameter space when their masses are similar to the mass of the dark matter. The effect of these coannihilations will lower the relic abundance [3].

We show in Fig. 2 the spin-independent $\eta_{R}$-nucleon elastic scattering cross section weighted by $\xi=\Omega_{\eta_{R}} / \Omega_{\text {Planck }}$ versus the $\eta_{R}$ mass. The color code of displayed points is the same as in Fig. 1 . The dark green solid line denotes the most recent upper bound from XENON1T [8]. The extended particle content characteristic of the Singlet + Triplet Scotogenic Model in principle allows for a viable scalar dark matter candidate in a wider region of masses, compared to the simplest scotogenic or Inert Higgs Doublet models. Nevertheless, because of current experimental constraints, most of the new allowed solutions with a relic abundance within the $3 \sigma$ C.L. measurement of the cold dark matter by the Planck collaboration [7, 9] lie in a tight vertical region around $m_{\eta_{R}} \sim 500-600 \mathrm{GeV}$. Lighter $\eta_{R}$ lead to viable dark matter, although under-abundant, hence it would require the existence of an additional dark matter candidate. We show in Fig. 3 the results of our numerical scan of the annihilation cross section (weighted by $\xi^{2}$ and by the correspondent branching ratio) versus the $\eta_{R}$ mass, for $\eta_{R}$ annihilating into $b \bar{b}$ (orange points), $\tau^{+} \tau^{-}$(dark cyan) and $W^{+} W^{-}$(dark red). Grey points are excluded by any of the constraints mentioned in [3]. Points in light red are solutions with relic abundance within the $3 \sigma$ range measured by Planck. In the same figure we also show the 95\% C.L. upper limits currently set by the Fermi-LAT satellite using $\gamma$-ray observations of Milky Way dwarf spheroidal satellite galaxies (dSphs), based on 6 years of data processed with the Pass 8 event-level analysis [12]. Solid lines assume annihilation into $b \bar{b}$ (orange), $\tau^{+} \tau^{-}$(dark cyan) and $W^{+} W^{-}$(dark red). Moreover, we show as a red dot-dashed curve the current upper limit obtained by H.E.S.S. using Galactic Center (GC) $\gamma$-ray data accumulated over 10 years [13], assuming a $W^{+} W^{-}$ channel and an Einasto dark matter density profile. Finally, we also depict sensitivity projections for 


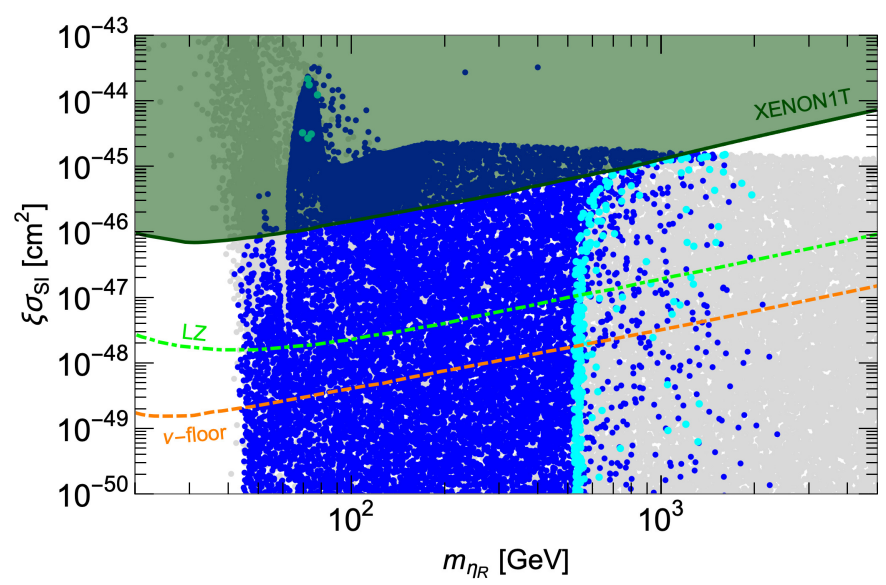

Figure 2: Spin-independent $\eta_{R}$-nucleon elastic scattering cross section versus the $\eta_{R}$ mass. Colour code as in Fig 1. The dark green line denotes the most recent upper bound from XENON1T [8]. The dashed orange line depicts the lower limit corresponding to the "neutrino floor" from coherent elastic neutrino-nucleus scattering (CEvNS) [10], while the green dot-dashed one stands for the projected sensitivity for LZ [11].

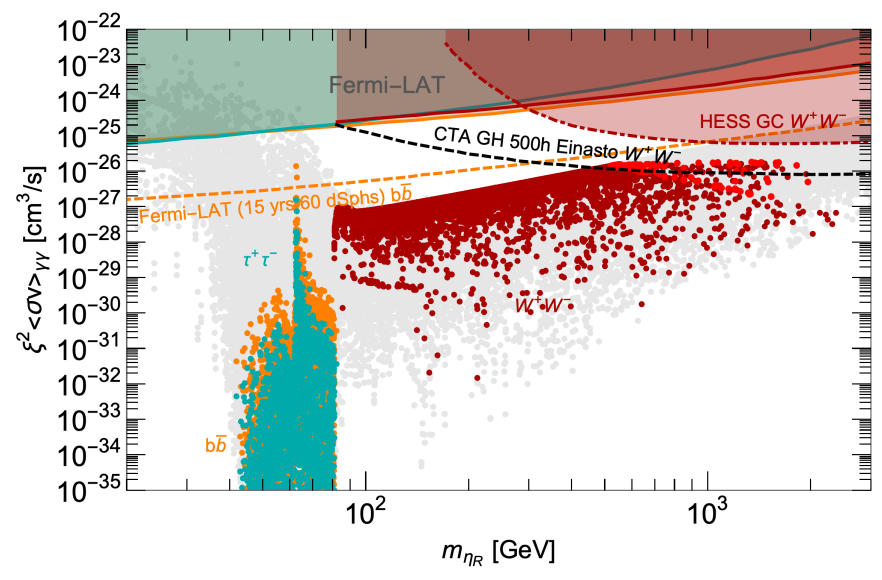

Figure 3: Predicted $\eta_{R}$ annihilation cross section into $\gamma$ rays - weighted by the relative abundance - for annihilations to $b \bar{b}$ (orange), $\tau^{+} \tau^{-}$(dark cyan) and $W^{+} W^{-}$(dark and light red) final states.

Fermi-LAT from a stacked analysis of $60 \mathrm{dSphs}$ and 15 years of data, in the $b \bar{b}$ channel [14] (dashed orange) and for CTA, for the Milky way galactic halo target, $W^{+} W^{-}$channel and an Einasto dark matter density profile [15]. Although current limits lie a couple of orders of magnitude above the predicted signals in this model, future data from Fermi-LAT and CTA offer promising prospects, eventually allowing one to test part of the parameter space both in the low $(\sim 70 \mathrm{GeV})$ as well as in the high ( $\gtrsim 500 \mathrm{GeV}$ ) mass regions.

Collider results We confront our scalar dark matter candidate with the latest data from particle colliders, in particular from the LHC run at $\sqrt{s}=13 \mathrm{TeV}$. We test the model using the CheckMATE 2 

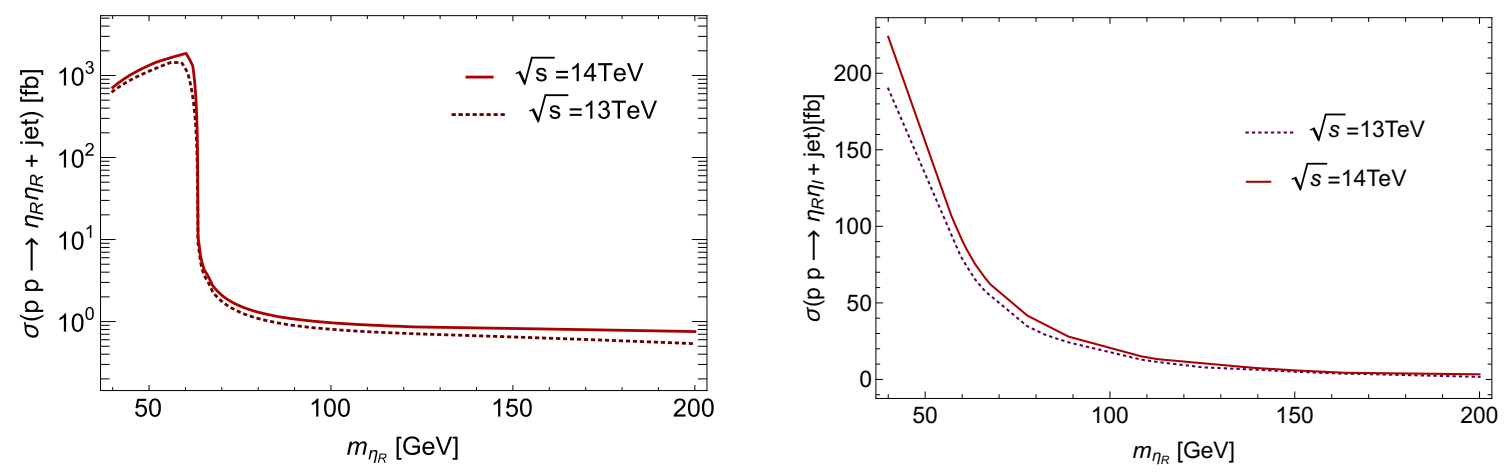

Figure 4: Cross sections of mono-jet signals at LHC $\sqrt{s}=13$ (14) TeV. The left panel shows the Higgs boson mediated events from $p p \rightarrow \eta_{R} \eta_{R}+$ jet. The maximum value of the cross section is $\sim 1400(1800) \mathrm{fb}$ for $\sqrt{s}=13$ (14) $\mathrm{TeV}$ respectively. The right panel is the Z-mediated process, $p p \rightarrow \eta_{R} \eta_{I}+$ jet, with peak contribution $\sim 190(220) \mathrm{fb}$.

collaboration tools $[16,18,19]$. In our scenario, the most promising signal is $\mathbb{E}_{T}+$ jet (mono-jet). In the following we will focus on mono-jet final states, arising from $p p \rightarrow \eta_{R} \eta_{R}+g$ and $p p \rightarrow \eta_{R} \eta_{R}+q$ processes. Besides Higgs mediation, the mono-jet signal can proceed also via Z-mediation if the mass difference between $\eta_{I}$ and $\eta_{R}$ is small. Therefore we also include the $p p \rightarrow \eta_{R} \eta_{I}+g$ and $p p \rightarrow \eta_{R} \eta_{I}+q$ processes. The benchmark point that was considered in our study is described in [3]. In Fig. 4 we present the production cross section for $\mathbb{E}_{T}+$ jet process at $\mathrm{LHC} \sqrt{s}=13$ (14) $\mathrm{TeV}$ calculated were the simulate events were calculated by Madgraph5 [21] . Since the relevant processes leading to these events are mediated by mainly the SM Higgs (left panel) and $Z$ boson (right panel), one has the characteristic peaks at $m_{\eta_{R}} \sim m_{h^{0}} / 2$ and at $m_{\eta_{R}} \sim m_{Z} / 2$ respectively, providing larger cross sections in these mass ranges. Therefore, the Higgs boson mediated processes are dominant up to $m_{\eta_{R}} \sim 60 \mathrm{GeV}$ and also contribute in the range $\sim[700-1400] \mathrm{fb}(13 \mathrm{TeV})$. In addition, $Z$-mediated processes complements the search for $p p \rightarrow \eta_{R} \eta_{R}+$ jet process at the LHC. For this mass range, the cross sections are $\sim[190-80] \mathrm{fb}$, while for dark matter masses between [65 - 200] GeV we have $\sim[70-5] \mathrm{fb}$, providing a sizeable contribution to the total mono-jet cross section, which could be within the LHC sensitivity. At $\sqrt{s}=14 \mathrm{TeV}$ the cross section increases by a few $\mathrm{fb}$. These results agree with expectations of other models, such as the Inert Higgs Doublet Model, whose contributions to this signal are very similar [22]. In summary, one sees that there are good prospects for probing the mono-jet signal at the LHC for dark matter masses up to $\sim 60 \mathrm{GeV}$.

Conclusions In our analysis we realize that there are good perspectives from future experiments to detect dark matter indirectly, if its mass is around $70 \mathrm{GeV}$ or higger than $500 \mathrm{GeV}$. We have also found that, for our benchmark point, there are good possibilities to detect the scalar dark matter from the enhanced mono-jet signal, if the dark matter mass is around $60 \mathrm{GeV}$. Nevertheless, deeper studies are necessary in order to compute direct detection signals at loop level.

\section{References}

[1] E. Ma, Phys.Rev. D73, 077301 (2006). DOI 10.1103/PhysRevD.73.077301 
[2] M. Hirsch, R. Lineros, S. Morisi, J. Palacio, N. Rojas, J. Valle, JHEP 1310, 149 (2013). DOI 10.1007/JHEP10(2013)149

[3] I.M. Ávila, V. De Romeri, L. Duarte, J.W. Valle, (2019)

[4] T. Hambye, F.S. Ling, L. Lopez Honorez, J. Rocher, JHEP 0907, 090 (2009). DOI 10.1088/ $1126-6708 / 2009 / 07 / 090$

[5] L. Lopez Honorez, C.E. Yaguna, JHEP 1009, 046 (2010). DOI 10.1007/JHEP09(2010)046

[6] M.A. Díaz, B. Koch, S. Urrutia-Quiroga, Adv.High Energy Phys. 2016, 8278375 (2016). DOI $10.1155 / 2016 / 8278375$

[7] P. Ade, et al., Astron.Astrophys. 594, A13 (2016). DOI 10.1051/0004-6361/201525830

[8] E. Aprile, et al., Phys.Rev.Lett. 121, 111302 (2018). DOI 10.1103/PhysRevLett.121.111302

[9] N. Aghanim, et al., (2018)

[10] J. Billard, L. Strigari, E. Figueroa-Feliciano, Phys.Rev. D89, 023524 (2014). DOI 10.1103/ PhysRevD.89.023524

[11] D.S. Akerib, et al., (2018)

[12] M. Ackermann, et al., Phys.Rev.Lett. 115, 231301 (2015). DOI 10.1103/PhysRevLett.115. 231301

[13] H. Abdallah, et al., Phys.Rev.Lett. 117, 111301 (2016). DOI 10.1103/PhysRevLett.117.111301

[14] E. Charles, et al., Phys.Rept. 636, 1 (2016). DOI 10.1016/j.physrep.2016.05.001

[15] B. Acharya, et al., Science with the Cherenkov Telescope Array (2017). DOI 10.1142/10986

[16] D. Dercks, N. Desai, J.S. Kim, K. Rolbiecki, J. Tattersall, T. Weber, Comput.Phys.Commun. 221, 383 (2017). DOI 10.1016/j.cpc.2017.08.021

[17] T. Sjostrand, S. Mrenna, P.Z. Skands, Comput.Phys.Commun. 178, 852 (2008). DOI 10.1016/ j.cpc.2008.01.036

[18] J. de Favereau, et al., JHEP 1402, 057 (2014). DOI 10.1007/JHEP02(2014)057

[19] M. Cacciari, G.P. Salam, G. Soyez, Eur.Phys.J. C72, 1896 (2012). DOI 10.1140/epjc/ s10052-012-1896-2

[20] A.L. Read, (2002), vol. G28, pp. 2693-2704. DOI 10.1088/0954-3899/28/10/313

[21] J. Alwall, et al., JHEP 1407, 079 (2014). DOI 10.1007/JHEP07(2014)079

[22] A. Belyaev, et al., Phys.Rev. D99, 015011 (2019). DOI 10.1103/PhysRevD.99.015011

[23] A. Belyaev, G. Cacciapaglia, I.P. Ivanov, F. Rojas-Abatte, M. Thomas, Phys.Rev. D97, 035011 (2018). DOI 10.1103/PhysRevD.97.035011 